RESEARCH ARTICLE

\title{
Clinical and Microbiological Impact of Inhaled Tobramycin Treatment on Cystic Fibrosis Patients with Pseudomonas aeruginosa
}

\author{
Francesca Dickhaus ${ }^{1}$, Mutasim Abu Hasan ${ }^{2}$, Elizabeth Tremblay $^{3}$, Kenneth Klinker $^{4}$, Kenneth Rand ${ }^{5,6}$ \\ Stacy G. Beal $\left.\right|^{5}$ \\ ${ }^{1}$ University of Florida College of Medicine, Gainesville, Florida, USA \\ ${ }^{2}$ University of Florida College of Medicine, Department of Pediatrics, Pediatric Pulmonary and Allergy Division, \\ Gainesville, Florida, USA \\ ${ }^{3}$ UF Health Shands Hospital, Department of Infection Control, Gainesville, Florida, USA \\ ${ }^{4}$ UF Health Shands Hospital, Department of Pharmacy Practice, Gainesville, Florida, USA \\ ${ }^{5}$ University of Florida College of Medicine, Department of Pathology, Immunology, and Laboratory Medicine, \\ Gainesville, Florida, USA \\ ${ }^{6}$ University of Florida College of Medicine, Department of Internal Medicine, Division of Infectious Diseases and \\ Global Medicine, Gainesville, Florida, USA
}

\begin{abstract}
Objective: Patients' with cystic fibrosis respiratory systems become colonized with pathogens as early as the first year of life. Some organisms are associated with a decline in pulmonary function and shortened survival, such as a mucoid strain of Pseudomonas aeruginosa (PA).

Methods: We conducted a four year retrospective chart review study examining 158 pediatric and adult cystic fibrosis patients treated at an academic, tertiary care institution to compare patients treated by different antimicrobial regimens.

Results: A higher proportion of mucoid PA study patients received alternate-monthly tobramycin therapy (83.3\%), compared to those with non-mucoid PA (62.5\%); although a large percentage of patients who had never had a mucoid PA infection were also receiving alternate-monthly tobramycin. Patients who had a mucoid PA infection had more hospitalizations and for longer periods of time than patients who had a non-mucoid PA infection; both mucoid and non-mucoid PA patients were hospitalized more often than patients who had never had a PA infection. We additionally found patients with mucoid PA infections to have strains that were either resistant or had intermediate resistance to tobramycin. Similar trends in resistance were not seen in patients who only received intermittent treatments with tobramycin.
\end{abstract}

Conclusions Our findings on increased rates of infection with both Aspergillus (in the alternate-monthly inhaled tobramycin group) and NTM (in the overall patient population) comparative to international averages were also interesting, and open the doors to future research regarding CF patients with these infections. $J$ Microbiol Infect Dis 2017; 7(4):178-185

Keywords: HIV, HCV, Genotype, Co-infection, Viral load

\section{INTRODUCTION}

Cystic fibrosis (CF) is the most common lethal genetic disorder among Caucasians, with an incidence of 1 in 3,000 live births and a carrier frequency of 1 in only 25 [1]. The disease is of autosomal recessive inheritance, and is caused by mutations in the CF transmembrane conductance regulator (CFTR) gene. This gene encodes a protein that functions in transepithelial ion transport and salt concentration regulation. There are more than 1900 reported CFTR mutations, and 1500 of these are considered to potentially cause CF. The pathogenic CF mutations can be divided into five classes which correspond to a large range in disease severity. Table 1 summarizes

Correspondence: Dr. Stacy G. Beal, University of Florida, College of Medicine, Department of Pathology, Immunology, and Laboratory Medicine, Florida, USA, E-mail: Stacygbeal@ufl.edu

Received: 20 March 2017 Accepted: 11 November 2017

Copyright (C JMID / Journal of Microbiology and Infectious Diseases 2017, All rights reserved 
some of the more common mutations seen in CF patients [2].

The primary organ system affected in cystic fibrosis patients is the respiratory system, although numerous other organ systems can also be involved. Thick mucus builds up in the airways and the lungs, leading to decreased lung function and an increased opportunity for infection. Because of this, the degree of morbidity and mortality in cystic fibrosis patients ultimately depends on the rate of deterioration of lung function [3].

Patients' respiratory systems become colonized with bacterial and fungal pathogens as early as the first year of life [4]. Therefore, antibiotics are a mainstay of CF treatment and have led to improved survival [5]. The most commonly cultured organisms from sputum or lower respiratory specimens are Pseudomonas aeruginosa (PA), Staphylococcus aureus, Haemophilus influenzae, non-tuberculous mycobacteria (NTM), and Aspergillus species [6]. Colonization with these organisms varies with the patient's age. For example, Haemophilus influenzae infections generally peak during the range of 2-5 years of age, whereas Aspergillus infections tend to increase with age. Additionally, some organisms are associated with a decline in pulmonary function and shortened survival, such as a mucoid strain of $P$. aeruginosa. The conversion of $P$. aeruginosa to its mucoid phenotype is thought to be driven by the unique environment within the lungs of CF patients [7]. Once such organisms have established a chronic infection with a mucoid strain, they are notoriously difficult to eradicate. This is due to the fact that the mucoid matrix contributes to the formation of a biofilm, which then allows the bacteria to resist phagocytosis, opsonization, and digestion. Since the 1970's, it has been evident that $P$. aeruginosa is most susceptible to the inhaled antibiotic tobramycin. Studies have shown that inhaled tobramycin treatment can delay the acquisition of mucoid $P$. aeruginosa in CF patients, thereby delaying decline in pulmonary function [5]. Tobramycin is a bactericidal aminoglycoside antibiotic that is effective against gram-negative microorganisms. It functions by irreversibly binding the $30 \mathrm{~S}$ subunit of the bacterial ribosome and disrupting protein synthesis, ultimately leading to alterations in cell membrane permeability and cell death.

Increasing antimicrobial resistance, however, is a worldwide concern for all patients and diseases. When these microorganisms are exposed to a drug for extended periods of time, they develop resistance through a variety of mechanisms, including enzyme modifications, lower outer membrane permeability, active efflux MexXY proteins, target modification, nonenzyme based mechanisms, and the accumulation of mutants. This is an especially important problem in CF patients, who have the type of mucus-coated environment in the lungs that allow bacteria to create biofilms and thrive, becoming resistant to many drugs.[8] Multidrug resistant Pseudomonas aeruginosa (MDR-PA) and methicillin-resistant Staphylococcus aureus (MRSA) are especially important concerns for patients with $\mathrm{CF}$.

CF has serious implications for the pulmonary system and eventually leads to significant increases in morbidity and mortality for the affected patients. Therefore, the aim of our study was to investigate the effect of mucoid Pseudomonas infection and alternate-monthly inhaled tobramycin treatment on CF status and other microbiological infections within the lungs.

\section{METHODS}

We conducted a retrospective chart review on all adult and pediatric patients with cystic fibrosis seen at UF Health Shands Hospital, an academic medical center, in a four-year study period, 6/1/11 until 5/31/15. Exclusion criteria included an age less than 6 years old at the start of the study period, status-post single or double lung transplant, or death within the study period. One hundred fifty-eight total patients were examined for inclusion in our study (Figure 1).

Of these, 32 of these were excluded on the basis of age, 6 received lung transplants, and 2 were deceased at the end of the study period. This study was approved by our Intuitional Review Board (\#IRB201500371). The data were collected exclusively from the electronic health record (Epic Systems (C) into a database using REDCap electronic data capture tools hosted at the University of Florida. Demographics included age, gender, and body mass index. Microbiological information included presence of 
PA infection, sensitivity or resistance of the PA to tobramycin, and any additional respiratory coinfections that occurred during the study period.

Patients were categorized as having no PA, non-mucoid PA, or mucoid PA based on morphologic characteristics seen in the microbiology laboratory.

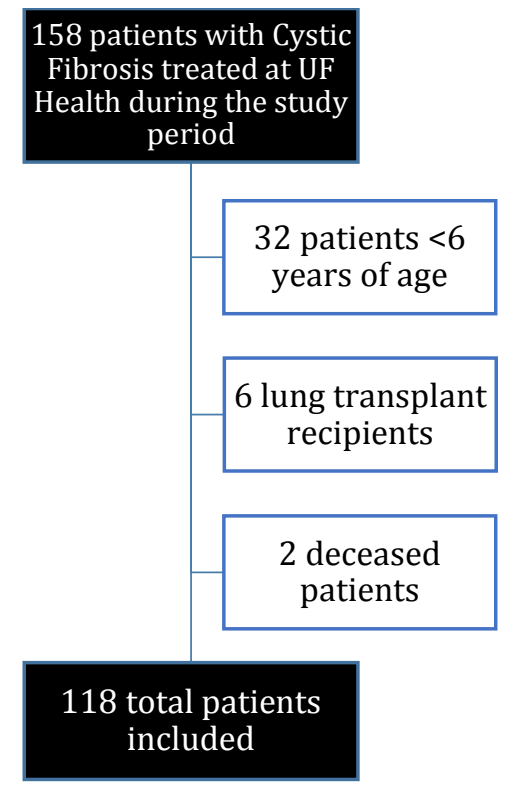

Figure 1: A total of 118 patients were included in our study.

If at any point during the study period the patient grew mucoid PA from a respiratory culture (sputum, bronchial wash, bronchoalveolar lavage, or tracheal aspirate), they were included in the mucoid PA group. In patients who had mucoid or non-mucoid PA, sensitivity of the organism to tobramycin was recorded. If any PA isolate was ever resistant during the study period, we placed them in the resistant group and recorded their first date of resistance. All organisms other than PA that were recovered in any of the respiratory cultures were also recorded.

Markers of CF disease status were also obtained. This included the CF gene mutation, pulmonary function testing results, presence of bronchiectasis, and total number of hospitalization days. The patient's use of inhaled tobramycin treatment was documented as never receiving tobramycin, received it intermittently, or received it on an alternate-monthly schedule (meaning he/she received the drug for one month, then did not receive it the next month, then did the following month, etc.). If they were ever on an alternate-monthly schedule, they were included in the alternate-monthly group. If they only received treatment intermittently, they were included in the intermittent group. For these patients, we recorded the number of intermittent treatments the patient received. Regardless of schedule, if the patient ever received tobramycin we recorded the first date of use. Lastly, we recorded the total number of hospitalizations and hospital days for each patient during the study period. We looked through the admission notes and only included hospitalizations for CF exacerbations specifically.

Tri-level comparative histograms were used to assess comparability in distribution between the three independent groups (no PA, non-mucoid $P A$, and mucoid PA). Further numerical analysis of similarities was completed using ANOVA and chi-squared tests.

Secondary statistical analyses were performed using SAS version 9.3 and Excel. Using mixed model regression we analyzed the percent predicted forced expiratory volume in 1 second (FEV1\%) values over time. A total of nine mixed models were used; one for each cross between tobramycin treatment group and Pseudomonas status group. The mixed models provided mean estimates of the slope and $y$-intercept for each, and when graphed together, the regression lines allowed us to compare the trends in FEV1\%.

\section{RESULTS}

Primary analyses of population demographics showed no significant difference ( $p$-value greater than 0.05 ) between any of the study groups (Table 2). A chi-squared test was used to compare the percentage of males in the no PA, non-mucoid PA, and mucoid PA groups $(p=0.6005)$ as well as similar rates of the homozygous F508delta gene mutations among the groups $(p=0.2955)$.

A total of $64 \%$ of our patients had a homozygous F508delta mutation. An additional $28 \%$ were heterozygous, possessing one F508delta mutation and one additional mutation (G542X, G551D, R553X, or other). Three percent of the patients were heterozygous with one G551D mutation and one other mutation. Additionally, 
$1 \%$ was heterozygous possessing one R553X 4\% had some other CF mutation (Figure 2). mutation and one other mutation. The remaining

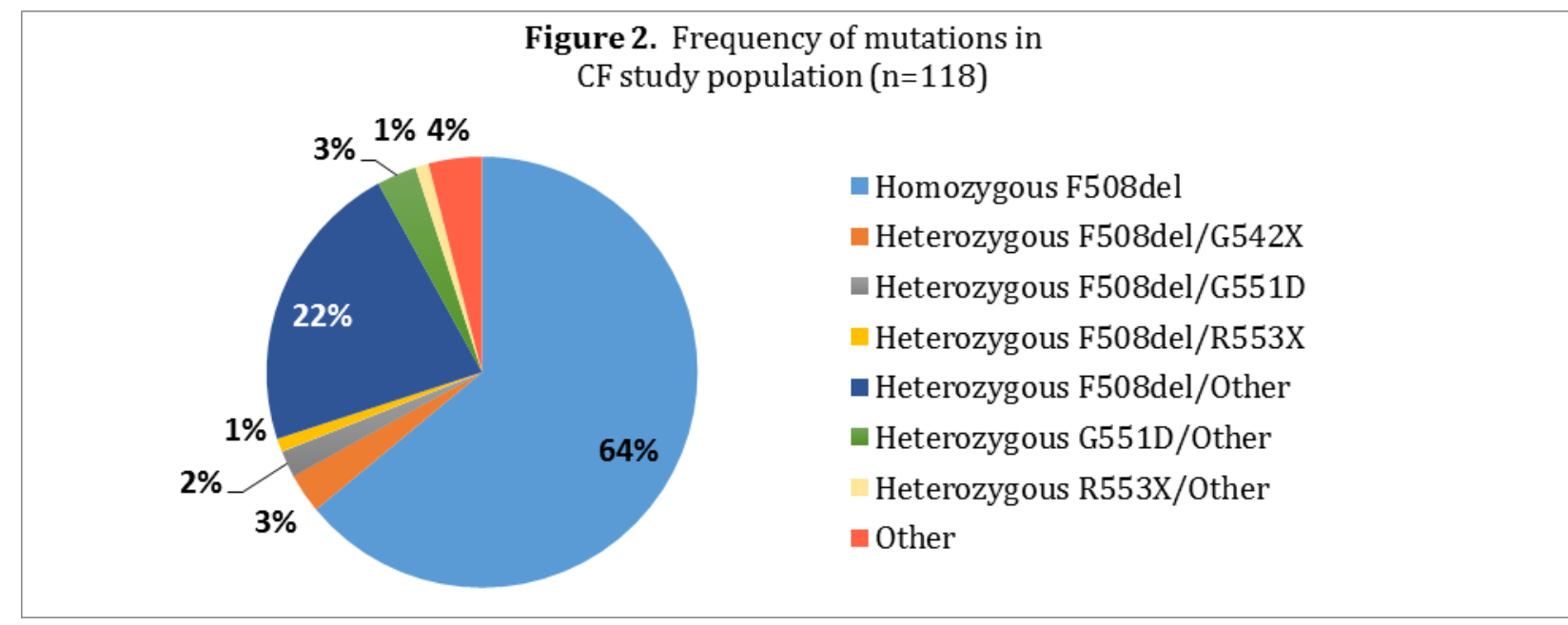

Figure 2: The most common mutation was homozygous F508del.

Seventy-four of our patients received alternatemonthly inhaled tobramycin treatment; 45 of these patients had a mucoid PA infection during the study period, 20 had a non-mucoid PA infection, and 9 had no PA infection. Twenty-four patients received intermittent tobramycin treatment; 7 of these patients had a mucoid PA infection, 9 had a non-mucoid infection, and 8 had no PA infection. The remaining 20 patients never received tobramycin treatment. Two of the 20 patients had a mucoid PA infection, 3 had a non-mucoid PA infection, and 15 never had a PA infection.

Of the 54 patients with a mucoid PA infection during the study period, $45(83.3 \%)$ received alternate-monthly inhaled tobramycin treatment, $7(13 \%)$ received tobramycin intermittently, and $2(3.7 \%)$ never received it at all. Of the 32 patients with a non-mucoid PA infection, 20 $(62.5 \%)$ received alternate-monthly inhaled tobramycin, $9(28.1 \%)$ received it intermittently, and $3(9.3 \%)$ never received it. Of the 32 patients with no PA infection during the study period, $9(28.1 \%)$ received alternate-monthly inhaled tobramycin, eight $(25 \%)$ received tobramycin intermittently, and 15 (46.9\%) never received tobramycin.

Sixteen patients with mucoid or non-mucoid PA received intermittent tobramycin 1,2 , or 4 times during the study period. One patient who received tobramycin 1 time was found to have
PA with resistance $(R)$ to tobramycin and 1 patient who received tobramycin 2 times was found to have PA with intermediate (I) resistance to tobramycin.

The average BMI of all 118 patients was 22.2. The average BMls for the patients in the no PA, non-mucoid PA, and mucoid PA groups were 21.5 (range: 15-29.6), 23.2 (range: 16.5-41.3), and 21.9 (range: 14.3-31.1) respectively.

Of all 118 patients, the average number of hospitalizations in the 4-year study period was 2.92 (range, 0-16) and the average total number of hospitalization days was 57.5 days (range, 0 - 719). Total hospitalization days were highest in patients with mucoid PA (average 70 days per patient), followed by non-mucoid PA (average 48 days), followed by no PA (average 43 days). The same trend was seen for number of hospitalizations: An average of 3.4 hospitalizations for patients with mucoid PA, 3.1 for non-mucoid PA, and 2.0 for patients with no PA. Patients with either type of PA had an average of 4.85 hospitalizations and 66.66 hospitalization days if they had I or R strains $(n=22)$ compared with 3.7 hospitalizations and 58.28 days if they had sensitive strains.

A total of 86 patients were found to have PA. One patient with non-mucoid PA who received intermittent tobramycin did not have susceptibilities performed on the isolate. A total of $27.7 \%$ and $12.31 \%$ of patients who received 
alternate-monthly tobramycin had resistant $(R)$ and intermediate (I) strains, respectively, of PA (mucoid or non-mucoid), compared with 6.3\% each for resistant and intermediate strains in patients who received intermittent tobramycin. There were no $\mathrm{R}$ or $\mathrm{I}$ isolates found for patients who never received tobramycin. This pattern held true when mucoid and non-mucoid PA patients are analyzed separately. For patients with mucoid PA, 28.9\% were resistant and $13.3 \%$ were intermediate for patients receiving alternate-monthly tobramycin, compared with $14.3 \% \mathrm{R}$ and $14.3 \%$ I for patients receiving intermittent tobramycin. Two patients with mucoid PA never received tobramycin and did not display any tobramycin resistance $(\mathrm{I} / \mathrm{R})$. For patients with non-mucoid PA, 25\% were resistant and $10 \%$ were intermediate for patients who received alternate-monthly tobramycin; whereas none of the 11 patients who received intermittent or no tobramycin was found to have intermediate or resistant strains.

Regardless of the type of tobramycin administration, mucoid strains were more often resistant and intermediate than non-mucoid strains: $14 / 54(25.9 \%)$ of mucoid strains vs. $5 / 32$ $(15.6 \%)$ of non-mucoid strains were resistant; $7 / 54(13.0 \%)$ of mucoid strains vs. $2 / 32(6.3 \%)$ of non-mucoid strains were intermediate.
Of all 118 patients, 86 (72.9\%) patients were found to have Staphylococcus aureus. Of these, $52(60.4 \%)$ were methicillin-resistant (MRSA) and $34 \quad(39.5 \%)$ were methicillin-sensitive (MSSA). This distribution was comparable between groups when stratified by mucoid PA, non-mucoid PA, and no PA. MRSA was found in $62.3 \%$ (33 of 53) patients with $S$. aureus who received tobramycin on an alternate monthly basis, compared with $57.9 \%$ (11 of 19) patients who received intermittent tobramycin and $57.1 \%$ (8 of 14) patients who never received tobramycin. Patients with mucoid PA, nonmucoid PA, or no PA all had similar rates of MRSA vs. MSSA.

Of 19 patients with PA (mucoid or non-mucoid) resistant to tobramycin, 14 also had $S$. aureus, of which $9(64.3 \%)$ were MRSA. Of 57 patients with PA sensitive to tobramycin, 45 had $S$. aureus; of which $28(62.2 \%)$ were MRSA.

Eighteen (15.3\%) patients had Aspergillus recovered in at least one respiratory culture. Of those, $13(72.2 \%)$ were in patients who received alternate-monthly tobramycin, compared to 4 $(22.5 \%)$ patients who received intermittent tobramycin and 1 (5.6\%) patient who never received tobramycin.

Table 2. Correlation of HCV positive cases with risk factors.

\begin{tabular}{lccc}
\hline Variables & No PA $\mathrm{n}(\%)$ & Non-mucoid PA n (\%) & Mucoid PA n (\%) \\
\hline $\mathrm{n}$ & 32 & 32 & 54 \\
Gender (male) & $16(50.0)$ & $20(62.5 \%)$ & $30(55.6 \%)$ \\
Age & $21.8(7.5-65.9)$ & $21.2(6.5-57.6)$ & $26.5(6.4-51.8)$ \\
BMI & $21.5(15.0-29.6)$ & $23.2(16.5-41.3)$ & $22.0(14.3-31.1)$ \\
Homozygous $\Delta$ F508 Genotype & $17(53.1)$ & $22(68.8)$ & $37(68.5)$ \\
\hline
\end{tabular}

Table 3. Mixed model analysis of Pseudomonas status by tobramycin treatment group

\begin{tabular}{lccc}
\hline Groups & Tobramycin Treatment & Estimate & p-value \\
\hline No PA & Never & -0.055 & 0.33 \\
& Intermittent & 0.480 & $<0.001$ \\
& Alt Monthly & -0.396 & $<0.001$ \\
\hline Non-Mucoid PA & Never & -0.228 & 0.0027 \\
& Intermittent & -0.163 & 0.12 \\
& Alt Monthly & -0.051 & 0.28 \\
\hline Mucoid PA & Never & -0.111 & 0.40 \\
& Intermittent & -0.064 & 0.44 \\
& Alt Monthly & -0.190 & $<0.001$ \\
\hline
\end{tabular}




\section{DISCUSSION}

The majority of our patients fell within one of three categories: those who had a non-mucoid or mucoid PA infection and had received alternate-monthly inhaled tobramycin treatment (20 patients and 45 patients, respectively) or patients who never had a PA infection and never received any tobramycin treatment (15 patients). Of those who had a mucoid infection, 83.3\% were on alternate-monthly inhaled tobramycin as compared to the $62.5 \%$ of patients with nonmucoid PA infection who also received alternate-monthly inhaled tobramycin. This is to be expected, since alternate-monthly inhaled tobramycin is the recommended treatment for CF patients with PA infections [9], particularly mucoid PA infections. If a patient never had a PA infection, there would be less of a need for them to be on tobramycin.

$\mathrm{BMI}$ is a surrogate measure of CF status and is lower in patients with worse CF disease [10]. Mucoid PA is often seen in more severe CF disease, we would expect patients with mucoid PA to have a lower average BMI. The lowest average BMI was seen in the group of patients who never had a PA infection, the middle average in the mucoid PA group, and the highest in the non-mucoid PA group. The lowest recorded $\mathrm{BMI}$ (14.3), however, belonged to a patient who had mucoid PA, which falls more in line with the trend that we anticipated. Although differences in ages between the groups were not statistically different, patients with no PA infection were younger (average 21.8) than patients with mucoid PA (average 26.5), which may be skewing the BMI results.

The results for the averages of the total number of hospitalizations and hospital days as related to mucoid status of the PA infection supported our hypothesis. The mucoid PA group had the highest averages for both hospitalizations and number of hospital days, followed by the nonmucoid PA group and no PA group respectively. This demonstrates the fact that patients with mucoid PA infections generally have more exacerbations of their disease, and thus have a worse CF status.

Our findings related to tobramycin resistance support our hypothesis that patients with mucoid PA infections have a worse CF status than patients with non-mucoid or no PA infections.
While the majority of patients in all three groups were sensitive to tobramycin, a larger percentage of the patients in the mucoid group grew either intermediate or resistant strains. Perhaps strains with the mucoid phenotype also contain genetic or phenotypic elements which make them more likely to become resistant to antibiotics. Another interesting result is that patients who were on alternate-monthly inhaled tobramycin treatment were more likely to have a resistant strain of PA (non-mucoid or mucoid). We conclude that resistance may develop with repeated exposure to the antibiotic.

Patients who received intermittent tobramycin treatment on 1, 2, or 4 occasions did not follow a similar trend. This may be because they were not exposed to the antibiotic as frequently as patients on alternate-monthly treatment; no trend in resistance was seen in this group. Therefore, it does not appear that receiving intermittent tobramycin on up to 4 occasions correlates with the presence of an $\mathrm{R}$ or I strain.

The large majority $(86 / 118)$ of our patients had a $S$. aureus coinfection at some point during the study period. There was a similar distribution of MRSA and MSSA among patients in the no, non-mucoid, and mucoid PA groups. There was also a similar distribution of MRSA and MSSA among patients who received tobramycin on an alternate-monthly basis, intermittent basis, or not at all. This indicates that both mucoid status of a PA infection and tobramycin administration likely do not have an effect on the resistance pattern of $S$. aureus. These results may exist due to the fact that $S$. aureus and PA infections are both acquired (transmission from patient-to-patient and the environment, respectively) and treated (linezolid/vancomycin and tobramycin, respectively) differently. These trends also may simply mirror the increased rates of MRSA within general hospital populations. MRSA and MSSA rates were also similar among the PA groups regardless of their resistance to tobramycin, which demonstrates that resistance mechanisms seem to be unrelated.

Of the 118 patients, 18 had Aspergillus infections at some point during the study period. Of these, 13 had been on alternate-monthly inhaled tobramycin, 4 received it intermittently, and 1 never received it. Additionally, 11 of the 18 also had mucoid PA, and 10 of these patients 
received alternate-monthly inhaled tobramycin. These results create two interesting questions. First, are the host factors that predispose patients to mucoid PA infections also setting them up for Aspergillus infection or colonization? Second, is the use of alternate-monthly inhaled tobramycin treatment to eliminate PA infections inadvertently selecting for Aspergillus?

Seventeen of the 118 patients (14.4\%) also had nontuberculous mycobacteria (NTM) growth at some point during the study period. The North American average for NTM growth in CF patients is generally $12 \%$ or lower. The average at UF Health is greater than this, which begs the question-how are the CF patients acquiring this organism? Historically, NTM was seen as nontransmissible from patient to patient. During one gene-mapping study at a United Kingdom Cystic Fibrosis center, 9 of 31 patients grew a genetically identical strain of NTM, and 2 more patients also shared a separate but identical strain [11]. This reinforces the thought that NTM may have some capabilities to transfer from patient-to-patient or may be acquired from a common source [12]. A previous study out of the University of Florida examined municipal water sources as a reservoir for NTM species. Higher prevalence in the water may be the reason for increased rates of NTM infection in our institution.

Our study had several limitations. First, a crosssectional study covering a four year time period may not be the best way to study a lifelong disease. Also, this study was entirely retrospective and all data were extracted from the electronic health record. The quality of our study data was dependent upon the completeness of the records by medical staff and the compliance of patients taking the medication as prescribed. Some of our study groups (mucoid PA with no tobramycin treatment) were very small, making comparisons between groups underpowered and inconclusive. Also, since UF Health is a lung transplant center, many CF patients are referred to our facility when their disease becomes more severe, requiring more advanced treatment. This lends itself to the fact that our study results are not generalizable to all CF patients and centers.

In conclusion, patients who had a mucoid PA infection at any point during the study period were more likely to be on alternate-monthly inhaled tobramycin treatment, although a large percentage of patients who had never had a mucoid PA infection were also likely to be on alternate-monthly inhaled tobramycin. Patients who had a mucoid PA infection were also more likely to be hospitalized (and for longer periods of time) than patients who had a non-mucoid PA infection, who were more likely to be hospitalized than patients who had never had a PA infection. Additionally, patients with mucoid PA infections on alternate-monthly therapy were found to have strains that were either resistant or had intermediate resistance to tobramycin, which leads us to conclude that resistance most likely develops in response to repeated use of the antibiotic (as observed in the alternatemonthly group). Similar trends in resistance were not seen in patients who only received intermittent treatments with tobramycin. Our findings on increased rates of infection with both Aspergillus (in the alternate-monthly inhaled tobramycin group) and NTM (in the overall patient population) comparative to international averages were also interesting, and open the doors to future research regarding CF patients with these infections.

\section{ACKNOWLEDGMENTS}

Conflict of interest: The authors declare no personal or financial conflict of interest.

Funding disclosure: Non to declare

\section{REFERENCES}

1. Strausbaugh SD, Davis PB. Cystic fibrosis: a review of epidemiology and pathobiology. Clin Chest Med 2007, 28:279-288.

2. De Boeck K, Zolin A, Cuppens H, Olesen HV, Viviani L. The relative frequency of CFTR mutation classes in European patients with cystic fibrosis. J Cyst Fibros 2014, 13:403-409.

3. Troxler RB, Hoover WC, Britton LJ, Gerwin AM, Rowe SM. Clearance of initial mucoid Pseudomonas aeruginosa in patients with cystic fibrosis. Pediatr Pulmonol 2012, 47:1113-1122.

4. Hauser AR, Jain M, Bar-Meir M, McColley SA. Clinical significance of microbial infection and adaptation in cystic fibrosis. Clin Microbiol Rev 2011, 24:29-70. 
5. Vazquez-Espinosa E, Giron RM, Gomez-Punter $\mathrm{RM}$, et al. Long-term safety and efficacy of tobramycin in the management of cystic fibrosis. Ther Clin Risk Manag 2015, 11:407-415.

6. Lipuma JJ. The changing microbial epidemiology in cystic fibrosis. Clin Microbiol Rev 2010, 23:299323.

7. Pritt B, O'Brien L, Winn W. Mucoid Pseudomonas in cystic fibrosis. Am J Clin Pathol 2007, 128:3234.

8. Barclay ML, Begg EJ, Chambers ST, Thornley PE, Pattemore PK, Grimwood K. Adaptive resistance to tobramycin in Pseudomonas aeruginosa lung infection in cystic fibrosis. J Antimicrob Chemother 1996, 37:1155-1164.

9. Lahiri T, Hempstead SE, Brady C, et al. Clinical Practice Guidelines From the Cystic Fibrosis Foundation for Preschoolers With Cystic Fibrosis. Pediatrics 2016, 137.

10. Gozdzik J, Cofta S, Piorunek T, Batura-Gabryel H, Kosicki J. Relationship between nutritional status and pulmonary function in adult cystic fibrosis patients. J Physiol Pharmacol 2008, 59 Suppl 6:253-260.

11. Bryant JM, Grogono DM, Greaves D, et al. Wholegenome sequencing to identify transmission of Mycobacterium abscessus between patients with cystic fibrosis: a retrospective cohort study. Lancet 2013, 381:1551-1560.

12. Qvist T, Pressler T, Hoiby N, Katzenstein TL. Shifting paradigms of nontuberculous mycobacteria in cystic fibrosis. In: Respir Res; 2014:41. vol 15.] 